\title{
First Hybrid Magnet for Neutron Scattering at Helmholtz-Zentrum Berlin
}

\author{
Peter Smeibidl, Mark Bird, Senior Member IEEE, Hartmut Ehmler, Iain Dixon, Member IEEE, Jochen Heinrich, \\ Matthias Hoffmann, Stephan Kempfer, Scott Bole, Jack Toth, Oleksandr Prokhnenko, Bella Lake
}

\begin{abstract}
Helmholtz-Zentrum Berlin operates two large scale facilities: the research reactor BER 2 and the synchrotron source for soft x-rays BESSY 2. This year HZB's neutron instrument suite around BER 2 has been strengthened by a unique high magnetic field facility for neutron scattering. Its main components are the High Field Magnet (HFM), the most powerful DC magnet for neutron scattering worldwide, and the Extreme Environment Diffractometer (EXED), the dedicated neutron instrument for time-of-flight technique. The hybrid magnet system is projected according to the special geometric constraints of analysing samples by neutron scattering in a high field magnet. Following our past experience only steady state fields are adequate to achieve the goals of the project. In particular inelastic scattering studies would virtually be excluded when using pulsed magnets. The new series-connected hybrid magnet with horizontal field orientation was designed and constructed in collaboration with the National High Magnetic Field Laboratory (NHMFL), Tallahassee, USA. With a set consisting of a superconducting cable-in-conduit coil and different resistive coils of conical shape, maximum fields between $26 \mathrm{~T}-31 \mathrm{~T}$ are possible with cooling power between $4 \mathrm{MW}$ $8 \mathrm{MW}$ for the resistive part. A series of commissioning activities of the magnet components and the technical infrastructure systems $(20 \mathrm{kA}$ power supply, water cooling and $4 \mathrm{~K}$ Helium refrigerator) was completed at HZB. The maximum field achieved with a $4 \mathrm{MW}$ resistive coil was $26 \mathrm{~T}$.
\end{abstract}

Index Terms-Neutron scattering, hybrid magnets, resistive magnets, superconducting magnets, $\mathrm{Nb}_{3} \mathrm{Sn}$, cable-in-conduit conductors.

\section{INTRODUCTION}

$\mathrm{N}$ NEUTRON SCATTERING is a powerful tool for studying structure and dynamics of matter over a wide range of length- and time-scales. Properties of neutrons, such as electrical neutrality, magnetic moment, and quasi-random variation of the scattering power as a function of atomic number, make it an ideal probe for many areas in modern physics, chemistry, and biology. Scattering experiments can benefit from high penetration depth of neutrons into many materials, neutron sensitivity to light elements, including

This work was supported in part by the German government (Ministry for Education and Research), the National Science Foundation (award DMR0603042) and the State of Florida.

P. Smeibidl is with the Helmholtz Centre Berlin, D-14109 Berlin, Germany, e-mail: peter.smeibidl@helmholtz-berlin.de.

H. Ehmler, J. Heinrich, M. Hoffmann, S. Kempfer, O. Prokhnenko and B. Lake are with the Helmholtz Centre Berlin, D-14109 Berlin, Germany.

M. Bird I. Dixon, S. Bole, J. Toth are with the National High Magnetic Field Laboratory, Tallahassee, FL 32310, USA. hydrogen, sensitivity to magnetism and many others.

Use of neutrons requires high effort and is only feasible at large scale facilities. For most neutron scattering applications neutrons are produced in research facilities by means of fission (in research reactors) or spallation (in acceleratordriven spallation neutron sources). Helmholtz-Zentrum Berlin (HZB), Berlin, Germany, operates the $10 \mathrm{MW}$ researchreactor BER II. The first $5 \mathrm{MW}$ reactor was built in 1972 and commissioned in 1973. In 1991 it was upgraded to $10 \mathrm{MW}$. At the same time a cold neutron source was installed. The reactor is primarily used for neutron generation in thermal and cold energy ranges centered around 25 and $10 \mathrm{meV}$, respectively. A number of neutron instruments uses neutron beams for scientific applications. The HZB neutron instrument suite includes instruments covering a broad range of experimental methods such as diffraction, small-angle scattering, reflectrometry, imaging, and spectroscopy. Special emphasis of the HZB is on neutron scattering under extreme conditions which include high pressure, high and low temperature, and high magnetic field and some of their combinations. In magnetic fields the HZB holds a world record of $17 \mathrm{~T}$ steady state (DC) field available for neutron experiments combined with temperatures as low as a few Kelvin (or $15 \mathrm{~T}$ for experiments in the milliKelvin temperature region). Neutron scattering in high magnetic fields has led to a number of important discoveries such as Bose-Einstein condensation in magnetic materials and field induced antiferromagnetic order in high temperature superconductors [1], [2]. However, research of condensed matter systems is bringing up an increasing number of questions which require experiments combining neutron scattering and magnetic fields above $17 \mathrm{~T}$. Therefore HZB started in collaboration with NHMFL an ambitious project of extending the magnetic field range significantly [3], [4]. One should mention that DC fields are essential for neutron scattering experiments, especially for those samples which need large data sets for good statistics. Pulsed magnets, which can reach fields of order of hundred Tesla, dependent on pulse length, have rather low duty cycle in neutron scattering experiments. During the available pulse length they can utilize only a negligible fraction of the neutrons that are available in the experiment.

\section{DESCRIPTION OF THE HIGH MAGNETIC FIELD FACILITY FOR NEUTRON SCATTERING}

In this contribution an overview of the HZB highmagnetic-field facility for neutron scattering is given. Its main components are the High Field Magnet (HFM), the most powerful DC magnet for neutron scattering in the world, and 
its neutron counterpart the Extreme Environment Diffractometer (EXED). The neutron scattering facilities at HZB are consisting of a reactor based neutron source with instruments for thermal and cold neutrons (see Fig. 1). A variety of different instruments is adapted to different scientific questions. A strong emphasis at HZB is given to experiments under extreme environments like low temperature, high pressure and high magnetic field.

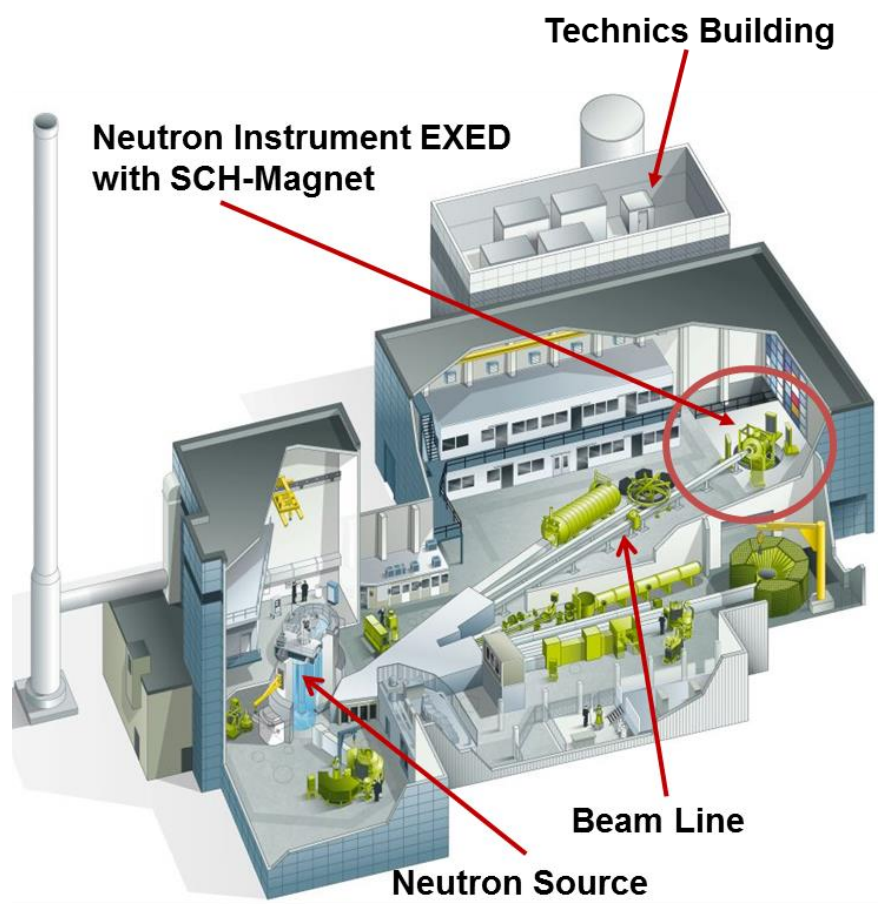

Fig. 1. Neutron scattering facilities at HZB.

Neutron source and neutron guide hall with the beam line to the High Field Magnet and the dedicated neutron instrument, the Extreme Environment Diffractometer beside the other neutron instrumentation. The building for the technical infrastructure of the magnet is in the background.

To reach very high DC fields, a completely different magnet technology has to be used compared to $15 \mathrm{~T}$ lowtemperature-superconductor magnets. This magnet technology is typically used at high magnetic field laboratories. It is now for the first time that a hybrid magnet was built for neutron scattering applications [5]. To combine the magnet and the neutron instrument successfully, they have to be adapted to each other. For that purpose, the magnet needs a geometry that is not fully optimal for achieving the highest fields while the neutron instrument has to compromise with a special design that takes into account the limited angular access for scattering experiments provided by the magnet.

\section{HYBRID MAGNET SYSTEM DESCRIPTION}

\section{A. Series-Connected Hybrid Magnet}

The High Field Magnet is a series-connected hybrid system with an outer superconducting coil and two inner normal-conducting coils. In collaboration of NHMFL and HZB this concept was implemented for the first time for design and construction of two systems, one at HZB and one at NHMFL. Both magnet systems are using the same concept
TABLE I

HYBRID MAGNET OPERATING SYSTEM PARAMETERS

\begin{tabular}{ll}
\hline \multicolumn{1}{c}{ Parameter } & \multicolumn{1}{c}{ Quantity } \\
\hline Magnet System Type & Series-Connected Hybrid \\
Central Field & $26 \mathrm{~T}(>30 \mathrm{~T})$ \\
Power Resistive Insert & $4 \mathrm{MW}(8 \mathrm{MW})$ \\
Warm Bore Diam. & $50 \mathrm{~mm}$ horizontal \\
Opening Angle & $300^{\circ}$ \\
Field Homogeneity & $<0.5 \%$ \\
& $(15 \mathrm{~mm} \times 15 \mathrm{~mm} \times 15 \mathrm{~mm}$ \\
& $\mathrm{cross} \mathrm{section} \mathrm{x} \mathrm{length)}$ \\
Operating Current & $20 \mathrm{kA}$ \\
Magnetic Field of Resistive Insert & $13 \mathrm{~T}-18 \mathrm{~T}$ \\
& $(4 \mathrm{MW} / 8 \mathrm{MW})$ \\
Coil Type of Resistive Insert & $\mathrm{CuZr} \mathrm{Florida} \mathrm{Bitter}$ \\
Magnetic Field of SC Coil & $13 \mathrm{~T}$ \\
Coil Type of SC Coil & $\mathrm{Nb}{ }_{3} \mathrm{Sn}$ cable-in-conduit \\
Stored Energy at max. Field & $43 \mathrm{MJ}$ \\
Inductance, resistive / supercon. coil & $3.8 \mathrm{mH} / 197.1 \mathrm{mH}$ \\
Cold Mass Weight & $\sim 6 \mathrm{t}$ \\
System Height & $\sim 5 \mathrm{~m}$ \\
System Weight & $\sim 25 \mathrm{t}$ \\
&
\end{tabular}

for the superconducting part but different resistive insert coils. The total field of the HZB system of $26 \mathrm{~T}$ with a $4 \mathrm{MW}$ insert coil has the potential to be increased to over $30 \mathrm{~T}$ with an upgrade in the power consumption to $8 \mathrm{MW}$. The system is designed for the special geometric constraints of performing neutron scattering experiments in a high field magnet. The main technical parameters are listed in Table I.

The inner resistive coil set is the first to have a conically shaped inner diameter to take advantage of the available space in the conical bore for the requirements of the neutron scattering experiment. It was developed at NHMFL to allow for scattering of neutrons upstream and downstream of the beamline towards detectors [6]. The superconducting coil is a 13 Tesla, $600 \mathrm{~mm}$ cold bore coil consisting of $\mathrm{Nb}_{3} \mathrm{Sn}$ cable-inconduit conductor (CICC) and weighs 6 tons (full cold mass including flanges, joints and piping). The design parameters are described in an internal project report to prepare for the discussion within a design review [7]. Before starting the procedure of ordering material an extended characterization and qualification campaign was performed which has included strand tests and full conductor tests for all three conductor types [8]-[12]. The final coil design includes 3 layers of highfield conductor, 3 layers of mid-field conductor and 12 layers of low-field conductor. The high-field cable pattern is $4 \times 3 \times 3 \times 3 \times 3$ with 4 as the final cable stage and 3 as the first cable stage with a total of $324 \mathrm{Nb}_{3} \mathrm{Sn}$ strands. The cable pattern of the mid-field conductor is $5 \times 4 \times 4 \times 3$ with 5 as the final cable stage and 3 as the first cable stage. This adds up to a total of 240 strands with 120 being composed of copper and 120 being composed of $\mathrm{Nb}_{3} \mathrm{Sn}$. Finally the low-field cable pattern is $4 \times 4 \times 4 \times 3$ with 4 as the final cable stage and 3 as the first cable stage and a total number of 192 strands with 128 being copper and 64 being $\mathrm{Nb}_{3} \mathrm{Sn}$. After completion of the conductor fabrication in Italy all conductors were transported to NHMFL [13].

Comparable to conductor development a qualification of components like joints and procedures like coil winding was performed prior to the start of coil winding [14], [15]. A structural analysis for the three cases normal operation, 
quench and fault of resistive insert coil verified that the conductor stress levels stay well below the limits [16]. The fabrication of the superconducting cable-in-conduit coil and the cold-mass assembly was completed at the National High Magnetic Field Laboratory (NHMFL), Tallahassee, USA at the end of 2013 [17], [18]. Finally the cold mass was transported to Europe for further assembly into the magnet cryostat together with a pair of $20 \mathrm{kA}$ current leads [19].

A sectional view of the magnet system is shown in Fig. 2. The inner resistive coil has a conically shaped inner diameter to conform to the bore. The resistive coils are series connected to a single superconducting coil with $\mathrm{Nb}_{3} \mathrm{Sn}$ CICC conductor.

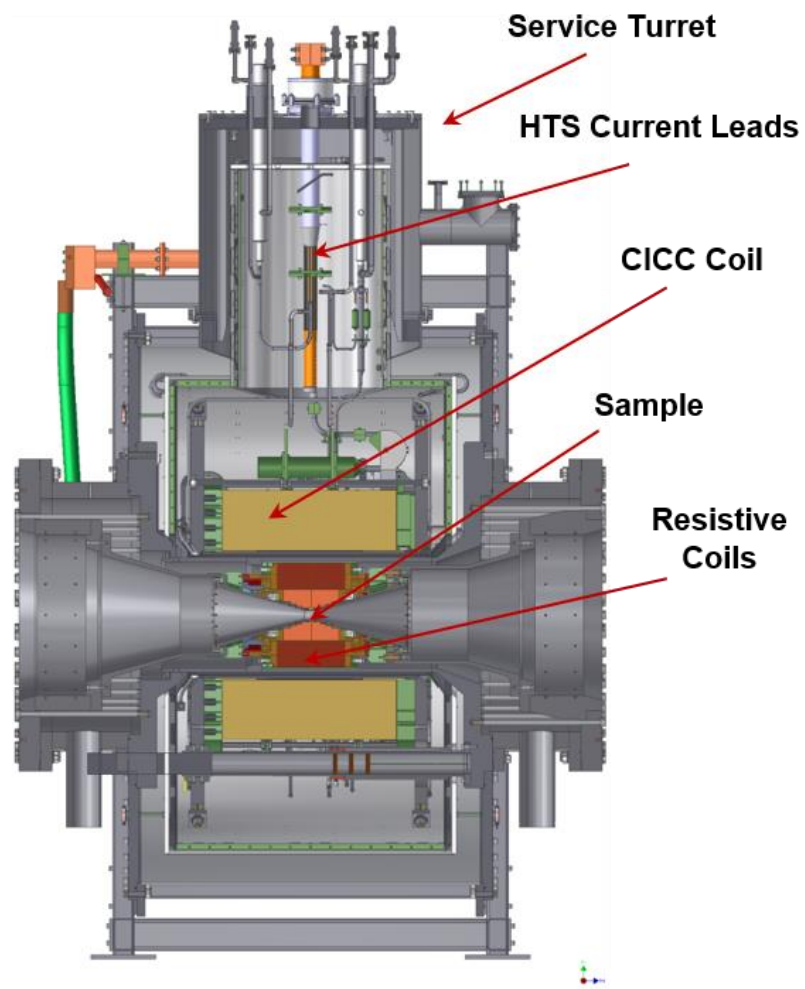

Fig. 2. Hybrid Magnet with superconducting and resistive coils. Sample position is in the center of the warm bore.

The entire magnet system has the bore horizontal so it can align with the neutron beam axis. In addition, the magnet system sits on an instrument table so it can rotate $+/-15^{\circ}$ for increased neutron scattering angle. All cryogenic and electrical utilities enter through a "service turret" on top of the magnet cryostat for interface with the supply systems. The thermal shields and the upper part of the current leads are cooled via the helium refrigerator down to nominally $40 \mathrm{~K}$.

\section{B. Coil Protection Systems}

The quench protection circuit consists of two DC breakers and a $100 \mathrm{~m} \Omega$ dump resistor. A Thyristor crowbar circuit beside the dump resistor ensures that the dump resistor is connected to the magnet only for the case of a fast ramp down. Together with the $197 \mathrm{mH}$ inductance of the CICC coil the protection circuit will discharge the coil with a time constant of two seconds and a maximum voltage of $2 \mathrm{kV}$ (please compare layout of the most important hardware components in Fig. 3).
For other failures than quench occurrence, the coil current can be ramped down through the resistive insert in "freewheeling mode of the power supply" with a time constant of 20 seconds or in "voltage-controlled mode" by the power supply. The current ramp rate from the power supply is adjustable and has an option for a "fast" ramp mode which is currently set to $50 \mathrm{~A} / \mathrm{s}$.

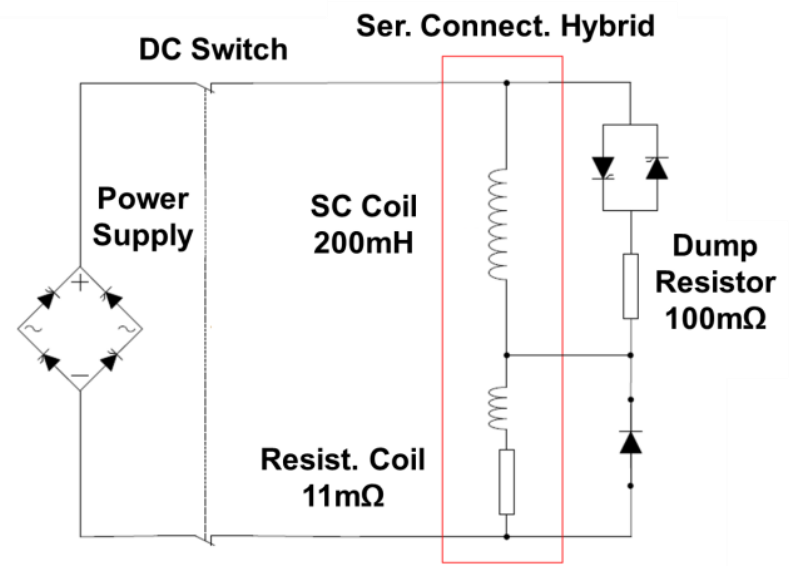

Fig. 3. Layout of power supply, series-connected magnet system and protection circuit.

Two independent quench detection units are used. The first quench detection system (QDS A) consists of voltage detection units. Each unit can either monitor a single voltage for observing the $\mathrm{NbTi}$ bus or the current leads or a difference of two voltages with a common mid-point for monitoring two adjacent coil layers. The voltage threshold for coil monitoring is $100 \mathrm{mV}$. The integration time varies between $50 \mathrm{~ms}$ and $100 \mathrm{~ms}$ for different detection units. These parameters ensure a fast quench detection and shutdown procedure. The coil temperature does not exceed $50 \mathrm{~K}$ in case of a quench.

The second unit (QDS B) represents a redundant detection for the CICC coil incorporating the differential signal from a co-wound wire, which follows all turns of the conductor. The voltage of this wire is compared to the coil voltage. Both QDS units are hard wire connected to the DC emergency breakers and to the power supply to ensure the reliability of the shutdown procedures.

The resistive magnet protection system (RMPS) monitors continuously several coil data as for example water flow, water pressure, water temperature, water conductivity and coil voltage (resistance). Limits for an emergency shut down are determined. The RMPS is also hard wire connected to the DC emergency breakers and to the power supply to ensure the reliability of the shutdown procedures.

\section{Sample Cryostat}

The sample is mounted in an independent cryostat to be inserted into the conical magnet bore. It's vacuum container fits precisely into the magnet bore to ensure the correct positioning of the sample with respect to the incoming neutron beam and the detectors for the scattered neutrons. The scheme in Fig. 4 shows the sample surrounded by the two radiation 
shields and the vacuum container sitting in the room temperature bore of the magnet.

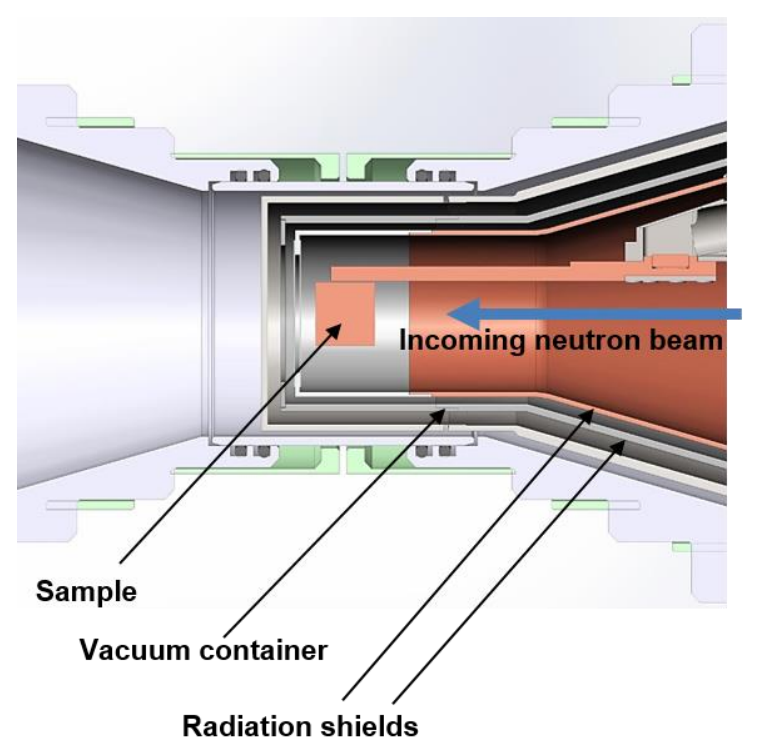

Fig. 4. Front end of the sample cryostat with the sample in the magnet center.

The cryostat provides sample temperatures down to about $0.5 \mathrm{~K}$ from two pumping stages for liquid ${ }^{3} \mathrm{He}$ and ${ }^{4} \mathrm{He}$. A pulse tube machine is used for precooling in the higher Kelvin temperature range.

\section{TECHNICAL INFRASTRUCTURE SYSTEMS}

The He-refrigerator system for the CICC coil and the $8 \mathrm{MW}$ power supply as well as the high pressure water circulation required to cool the heat loss from the resistive insert magnet, including the necessary cooling towers and the water treatment plant were constructed using standardised industrial components in a separate technical building for the HFM beside the Neutron Guide Hall. The layout in Fig. 5 shows the placement of the components in the technics building.

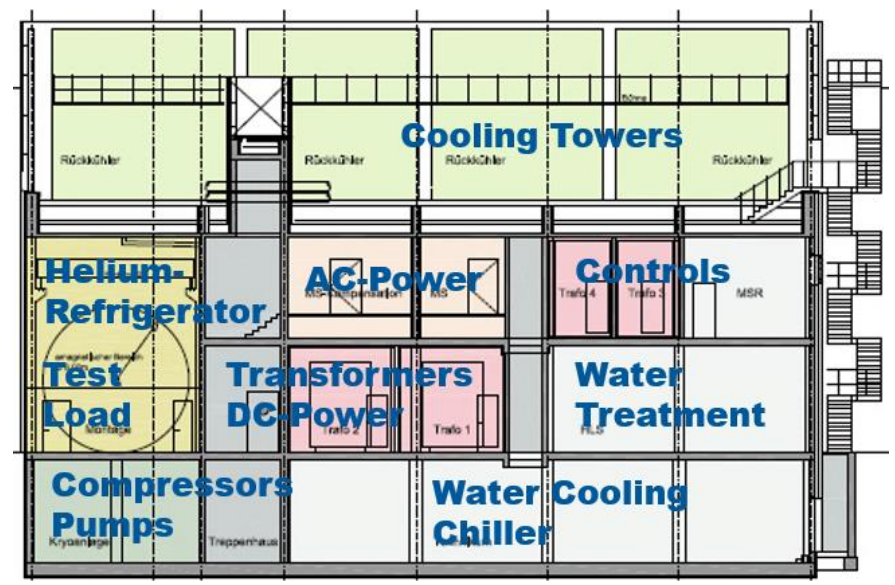

Fig. 5. Technics building with all components for operation of the hybrid magnet.

A central control system to combine the local control units of the three big components and to provide a user terminal for the magnet operation completes the technical infrastructure installations.

\section{A. Power Supply}

The power supply integrates all components from the $10 \mathrm{kV}$ switch gear to the current connectors at the magnet. The main components are transformers, a 24 pulse thyristor rectifier system, a water cooled Al busbar system, DC emergency breakers, a dump resistor and a pair of water cooled flexible leads to allow rotation of the magnet.

The power supply provides a maximum current $20 \mathrm{kA}$ at $400 \mathrm{~V}$ with a current ripple of several $10 \mathrm{ppm}$.

\section{B. Helium Refrigerator}

The He-refrigerator system was specially designed for the cooling of the superconducting coil in a closed pure gas loop. The cooling is performed with supercritical $\mathrm{He}$ at a minimum pressure of 2.7 bar. In addition the system provides $40 \mathrm{~K}$ Helium gas for the cooling of the upper part of the HTS current leads and for the radiation shields in the magnet cryostat. The refrigerator runs continuously. The CICC coil is kept cold independent from the magnet operation.

\section{Water Cooling}

The water cooling system is a standard setup with a high pressure ( $16 \mathrm{bar})$, high purity $(0.1 \mu \mathrm{S} / \mathrm{cm}$ conductivity) magnet loop connected via a heat exchanger to a second loop with cooling towers and a chiller.

\section{Control System}

The Siemens S7 units of the Power supply, water cooling and He-refrigerator are connected to a central control system. The system monitors about 500 parameters which are related to the magnet or to components in the technics building. Limits are determined for important control parameters to ensure safe shutdown procedures. In addition the control system provides a user terminal. It accepts the input from the neutron instrument users, checks all control parameters of the system and starts a field ramp if all parameters are within the allowed limits. The system changes into a safe status if failure conditions are present, which have to be checked and can be released only by control room staff members.

\section{NEUTRON INSTRUMENTATION}

\section{A. Extreme Environment Diffractometer EXED}

The EXED instrument is currently a time-of-flight (TOF) instrument optimized for diffraction and small angle neutron scattering (SANS) under restricted geometrical conditions imposed by the magnet geometry, with $30^{\circ}$ neutron access in forward and backward scattering [20]. To achieve that it utilizes polychromatic (time-of-flight) technique which is not very typical for instruments on a continuous (reactor) source. Most of the instruments on reactor sources are monochromatic instruments exploiting a high time-averaged flux of the source. They use single incident neutron wavelength while scanning over the range of scattering angles to cover the requested scattering length according to the Bragg's law. This approach, however, would be ineffective for EXED in combination with 
HFM because of the restricted scattering angle. But using a range of wavelengths instead allows to compensate for the limited angle. In order to provide access to a broad wavelength range EXED is equipped with a so-called bispectral extraction system which allows neutrons from both, the thermal and the cold moderator entering the same neutron guide.

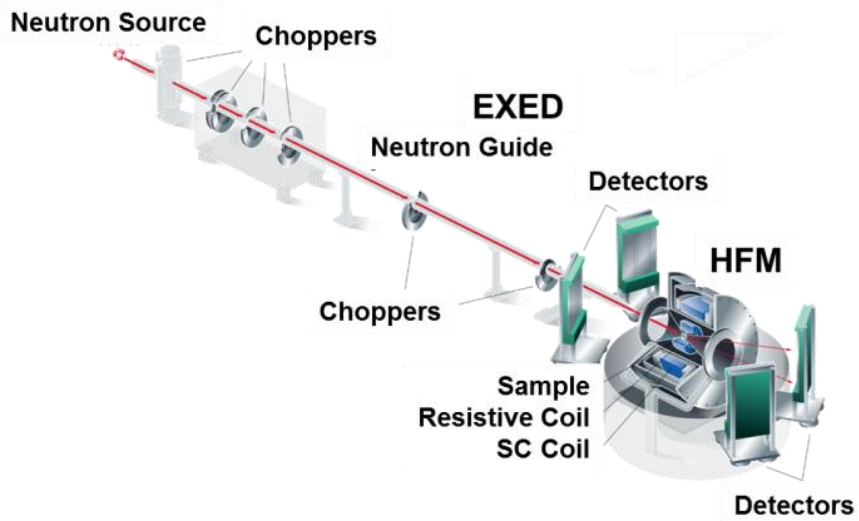

Fig. 6. Schematic layout of EXED showing the neutron source and main instrument components: the neutron guide, chopper cascade, and the HFM surrounded by detector banks. The picture is not to-scale, and the bi-spectral extraction system is not shown.

EXED has to cope with the complexity of the whole magnet project that comprises high effort for an extended technical infrastructure and therefore high investments. This led to plans for an upgrade of EXED with an optimum choice of different neutron experiments. In addition to improving the diffraction performance (signal-to-noise ratio and full angular coverage), it is especially important to complement the instrument portfolio by inelastic neutron scattering capabilities in the form of a direct time-of-flight (TOF) spectrometer. This unique experimental setup will play a major role in high-field neutron diffraction and spectroscopy. It will also strengthen the leading position of HZB in high magnetic field neutron scattering as it will offer by far the highest steady magnetic field for neutron research.

The implementation of inelastic scattering capabilities with its weak scattering cross sections imply the need for further optimization of signal strength and low background conditions. The former is achieved by enhancing the flux at the sample using a novel focusing guide while the latter is provided by means of a shielded and evacuated detector chamber. Therefore we are planning an upgrade of the neutron guide and an upgrade of the detector system beside the most important issue, the installation of an additional chopper close to the sample position. It is foreseen to complete this upgrade before end of 2016 .

\section{B. Scientific Opportunities}

What kind of experiments will become possible on EXED in combination with HFM, is a key question for the project. Since the magnet is permanently installed on one instrument, this instrument should be capable of performing all important experimental methods. This approach is not typical for neutron instruments being optimized for one class of experiments only. In order to cope with a broad range of scientific tasks, EXED will operate after the upgrade completion in three modes: diffraction, small angle scattering, and spectroscopy. In diffraction mode the instrument is suited for conventional crystallographic tasks, in particular studying static magnetic order in materials as function of applied magnetic field. Small angle neutron scattering mode extends the accessible d-range beyond $500 \AA$ enabling studies of matter in nanoscale range such as e.g. vortex state in type-two superconductors. Finally, the spectroscopy mode will make energy-resolved measurements on EXED possible. They will open a large area of research related to studying dynamics in materials with magnetic degrees of freedom.

\section{COURSE OF SYSTEM COMMISSIONING}

After completion of the last assembly tasks the magnet system was connected to all infrastructure facilities and to the data acquisition and control systems and the testing was started with the resistive coil alone.

All operating data (coil stress and cooling performance) were in agreement with our expectations. On $23^{\text {rd }}$ June 2014 the full field of $13.1 \mathrm{~T}$ at $20 \mathrm{kA}$ and $4 \mathrm{MW}$ was attained for the first time.

The next step was the connection of the superconducting coil to the He refrigerator via the flexible transfer lines and the cool down of the $6 \mathrm{t}$ cold mass to $4 \mathrm{~K}$. The base temperature was reached on $8^{\text {th }}$ Aug 2014. Since then the full hybrid system commissioning was performed. At first a complete check of all interfaces and data acquisition systems of the central control system was executed. The checks of the system safety include the interlocks of all peripheral systems for the operation of the power supply and the adjustment and fine tuning of the coil protection systems for the resistive and superconducting coils. With increasing system current the performance data have to be verified and for each limit overrun of operating data the emergency shutdown procedures have to be tested. The impact on the operation of all system components for the magnet and the technical infrastructure needs to be checked.

After 7.5 years of design and construction and after a series of commissioning activities of the technical infrastructure and the High Field Magnet the system has reached on $16^{\text {th }}$ Oct 2014 for the first time its maximum current of $20 \mathrm{kA}$ and full field of $26.2 \mathrm{~T}$.

Finally the magnet was transported from the assembly and test site in the technics building into the neutron guide hall and installed on the neutron instrument EXED. The final commissioning of magnet and neutron instrument together was successfully completed in June 2015.

Since then first user experiments were started. The challenge now is to prove the capability of all components of this complex facility for a reliable operation in long term user experiments.

\section{CONCLUSION}

HZB, in collaboration with the National High Magnetic Field Laboratory, Tallahassee, FL, USA, has recently finalized a project that combines a dedicated neutron scattering instrument with a horizontal magnet with tapered cones at 
both ends. The HFM-EXED facility is a unique combination of a high field hybrid magnet and neutron scattering instrument. It offers the highest static magnetic fields for neutron scattering in the world. The field is much higher than those available elsewhere and will remain so for many years.

\section{ACKNOWLEDGMENT}

The authors are deeply indebted to the many people at the NHMFL and HZB whose competence, confidence and tireless efforts enabled this project to be a success. We also greatly appreciate numerous discussions and support from our advisors from the ITER project and the community of European High Magnetic Field Laboratories.

\section{REFERENCES}

[1] B. Lake et al., "Antiferromagnetic order induced by an applied magnetic field in a high temperature superconductor", Nature vol. 415, p. 299 302, 2002.

[2] Ch. Rüegg et al., "Bose-Einstein condensation of the triplet states in the magnetic insulator $\mathrm{TlCuCl}_{3}$ " Nature vol. 423, p. 62 - 65, 2003.

[3] M. D. Bird et al., "The NHMFL hybrid magnet projects," IEEE Trans. Appl. Supercond., vol. 19, pp. 1612 - 1616, 2009.

[4] A. Bonito-Oliva et al., "Development of the Superconducting Outserts for the Series-Connected Hybrid Program at the NHMFL", IEEE Transactions on Appl. Supercond., vol. 18, 2008, p. 529 - 532

[5] P. Smeibidl, A. Tennant, H. Ehmler, M. D. Bird, "Neutron Scattering at highest magnetic fields at the Helmholtz Centre Berlin", J. Low Temp. Phys, vol. 159, pp. $402-405,2010$.

[6] J. Chen et al., "Conical Insert Design for the HZB Series-Connected Hybrid", IEEE Transactions on appl. Supercond., vol. 22(3), 2012, Art. ID. 4301304.

[7] I. R. Dixon et al., "Series-Connected Hybrid Outsert Coil Design for the Helmholtz Centre Berlin", Internal Project Report, Doc. ID HFM-MAGR00721.0.

[8] I. R. Dixon et. al., "Electromagnetic Cycling and Strain Effects on $\mathrm{Nb}_{3}$ Sn Cable-in-Conduit Conductors with Variations in Cabling Design and Conduit Material Properties", IEEE Transactions on Appl. Supercond., vol. 19(3), 2009, p. 1462 - 1465.

[9] J. Lu et. al, "Characterization of High $\mathrm{T}_{\mathrm{C}} \mathrm{Nb}_{3} \mathrm{Sn}$ Strands for the SeriesConnected Hybrid Magnet", IEEE Transactions on Appl. Supercond. vol. 19(3), 2009, p. 2615 - 2618.

[10] I. Dixon et. al., "Current Sharing and AC Loss Measurements of a Cable-in-Conduit Conductor with $\mathrm{Nb}_{3} \mathrm{Sn}$ Strands for the High Field Section of the Series-Connected Hybrid Outsert Coil", IEEE Transactions on Appl. Supercond., vol. 19, 2009, p. 2466 - 2569.

[11] I. Dixon et. al., "Qualification Measurements of the Mid-Field and LowField CICC for the Series Connected Hybrid Magnets with Effects of Electromagnetic Load Cycling and Longitudinal Strain", IEEE Transactions on Appl. Supercond., vol. 20(3), 2010, p. 1459 - 1462.

[12] J. Lu et. al, "Quality Assurance Tests of $\mathrm{Nb}_{3} \mathrm{Sn}$ Wires for the SeriesConnected Hybrid Magnets", IEEE Transactions on Appl. Supercond., vol. 21(3), 2011, p. 2571 - 2574.

[13] I. R. Dixon et. al. " $\mathrm{Nb}_{3} \mathrm{Sn}$ Cable-in-Conduit Conductor Fabrication for the Series-Connected Hybrid Magnets", IEEE Transactions on Appl. Supercond., vol. 22(3), 2012, Art. ID. 4301304.

[14] H. Bai et al., "Joint Design and Test for the SCH", IEEE Transactions on Appl. Supercond., vol. 21(3), 2011, p. 2122 - 2125.

[15] T. Adkins et al., Series-Connected Hybrid Outsert Coil Winding Hardware Design", IEEE Transactions on Appl. Supercond., vol. 21(3), 2011, p. $2114-2117$

[16] Y. Zhai, et. al., "Mechanical Analysis of the Superconducting Outsert for the Series-Connected Hybrid Magnets", IEEE Transactions on Appl. Supercond., vol. 19(3), 2009, p. 1608 - 1611.

[17] I. R. Dixon et al., „Fabrication Progress of the Outsert Coils of the Series-Connected Hybrid Magnets", IEEE Transactions on Appl. Supercond., vol. 23(3), 2013, Art. ID. 4300204.

[18] I. R. Dixon et. al. "Reaction Heat Treatment and Epoxy Impregnation of a Large $\mathrm{Nb}_{3} \mathrm{Sn}$ CICC Coil for a Series-Connected Hybrid Magnet",
IEEE Transactions on Appl. Supercond., vol. 24(3), 2014, Art. ID. 43100505.

[19] I. Dixon et al., "Final Assembly of the Helmholtz Zentrum Berlin Series-Connected Hybrid Magnet System", IEEE Transactions on Appl. Supercond., vol. 25(3), 2015, Art. ID. 4300204.

[20] O. Prokhnenko, W-D. Stein, H-J. Bleif, M. Fromme, M. Bartkowiak, T. Wilpert, "Time-of-Flight Extreme Environment Diffractometer at the Helmholtz-Zentrum Berlin”, Rev. Sci. Instr., vol. 86, 2015, Art. ID. 033102 . 